\title{
Wiener chaos and uniqueness for stochastic transport equation
}

\author{
Mario Maurelli ${ }^{\mathrm{a}}$ \\ ${ }^{a}$ Scuola Normale Superiore, Piazza dei Cavalieri 7, 56126 Pisa, Italy \\ Received *****; accepted after revision +++++ \\ Presented by
}

\begin{abstract}
We prove a uniqueness result for the stochastic transport linear equation (STLE), without any $W^{1,1}$ or $B V$ hypothesis on the coefficient, which is needed for the corresponding deterministic equation. We use Wiener chaos decomposition to pass from the STLE to a deterministic second-order transport equation with uniqueness property. To cite this article: M. Maurelli, C. R. Acad. Sci. Paris, Ser.??.

Résumé

Chaos de Wiener et unicité pour l'équation de transport stochastique. On prouve un résultat d'unicité pour l'équation de transport linéaire stochastique (STLE), sans aucune hypothèse de type $W^{1,1}$ ou $B V$ sur le coefficient, qui est nécessaire pour l'équation déterministe correspondante. On utilise la décomposition en chaos de Wiener pour passer de la STLE à une équation de transport du second ordre déterministe avec la propriété d'unicité. Pour citer cet article : M. Maurelli, C. R. Acad. Sci. Paris, Ser.??.
\end{abstract}

\section{Introduction}

On a probability space $(\Omega, \mathcal{F}, P)$, we consider the stochastic transport linear equation (STLE)

$$
d u_{t}+b \cdot \nabla u_{t} d t+\sum_{k=1}^{d} \partial_{k} u_{t} \circ d W_{t}^{k}=0
$$

where $W$ is a $d$-dimensional Brownian motion with respect to a certain right-continuous completed filtration $\left(\mathcal{F}_{t}\right)_{t}, b$ is a deterministic field on $\mathbb{R}^{d}, u$ is a random function defined on $[0, T] \times \mathbb{R}^{d}$.

The classical theory for the deterministic transport linear equation (i.e. without the stochastic integral), developed by DiPerna-Lions ([6]) and Ambrosio ([1]) and based on renormalized solutions, gives existence

Email address: mario.maurelli@sns.it (Mario Maurelli). 
and uniqueness in the class of weak $L^{\infty}$ solutions under hypotheses (a bit simplified for brevity) $b \in$ $L^{\infty}\left(\mathbb{R}^{d}\right) \cap B V_{\text {loc }}\left(\mathbb{R}^{d}\right)$ and $\operatorname{div} b \in L^{\infty}\left(\mathbb{R}^{d}\right)$; such hypotheses cannot be relaxed too much. As Flandoli et al. have shown, the introduction of noise allows some improvements: existence and uniqueness hold asking $b$ Hölder continuous, $\operatorname{div} b \in L^{q}$ for $q>2([7])$ or $b \in L^{\infty}\left(\mathbb{R}^{d}\right) \cap B V_{l o c}\left(\mathbb{R}^{d}\right), \operatorname{div} b \in L^{1}\left(\mathbb{R}^{d}\right)([3])$.

In this article we prove a new uniqueness result for STLE; the direct hypotheses on $b$, namely $b \in$ $L^{\infty}\left(\mathbb{R}^{d}\right)$, are weaker than those of the previous (stochastic) results, with the price of using the Brownian filtration and of a stronger integrability assumption on divb. Our approach is based on Wiener chaos decomposition, which allows to reduce the STLE to the associated Kolmogorov equation (namely the equation obtained by taking the expectation), which has uniqueness property for the Laplacian term. This approach was inspired by the article [9] by Le Jan and Raimond, where Wiener chaos is used to prove uniqueness for generalized stochastic flows.

Note that this result is false in the deterministic case: we cite two counterexamples in the end. Relations with pathwise uniqueness for the corresponding SDE are briefly recalled.

\section{Wiener chaos decomposition and the main result}

We define the operators $B f=b \cdot \nabla f, D_{k} f=\partial_{k} f, K f=\operatorname{tr}\left(D^{2} f\right)=\Delta f$; we use $B^{*}, D_{k}^{*}, K^{*}$ for their formal adjoint operators in $L^{2}\left(\mathbb{R}^{d}\right)$.

Definition 2.1 If $u_{0} \in L^{p}$, a weak $L^{p}$ solution of (1) on $\mathbb{R}^{d}$ is a function $u \in L^{p}\left([0, T] \times \mathbb{R}^{d} \times \Omega\right)$, progressively measurable with respect to $\left(\mathcal{F}_{t}\right)_{t}$, such that, for every $\phi \in C_{c}^{\infty}\left(\mathbb{R}^{d}\right), u_{r} B^{*} \phi$ is in $L^{1}\left([0, T] \times \mathbb{R}^{d}\right)$ for a.e. $\omega \in \Omega, u_{r} D^{*} \phi$ is a continuous semi-martingale with respect to $\left(\mathcal{F}_{t}\right)_{t}$, and it holds

$$
\int_{\mathbb{R}^{d}} u_{t} \phi d x=\int_{\mathbb{R}^{d}} u_{0} \phi d x+\int_{0}^{t} \int_{\mathbb{R}^{d}} u_{r} \frac{1}{2} K^{*} \phi d x d r-\int_{0}^{t} \int_{\mathbb{R}^{d}} u_{r} B^{*} \phi d x d r-\sum_{k=1}^{d} \int_{0}^{t} \int_{\mathbb{R}^{d}} u_{r} D_{k}^{*} \phi d x d W_{r}^{k} .
$$

Equation (2) is the weak formulation of (1), where we have used the link between Stratonovich integral and Ito integral (since $u_{r} D^{*} \phi$ is a continuous semi-martingale).

Definition 2.2 Let $P$ be the Wiener measure on the space $\Omega=C\left(\left[0,+\infty[, \mathbb{R})^{d}\right.\right.$. We write $\Delta_{n}(T):=$ $\left\{\left(t_{1}, \ldots t_{n}\right) \mid 0 \leq t_{1} \leq \ldots t_{n} \leq T\right\}$. For $f \in L^{2}\left(\Delta_{n}(T)\right)^{n d}$ we define the stochastic iterated integral

$$
\int_{\Delta_{n}(T)} f(r) d^{n} W(r):=\sum_{k_{1}, \ldots k_{n}} \int_{0}^{T} \int_{0}^{r_{n}} \ldots \int_{0}^{r_{2}} f_{k_{1}, \ldots k_{n}}\left(r_{1}, \ldots r_{n}\right) d W^{k_{1}}\left(r_{1}\right) \ldots d W^{k_{n}}\left(r_{n}\right) .
$$

The integral above is an injective isometry between the Hilbert spaces $L^{2}\left(\Delta_{n}(T)\right)^{n d}$ and $L^{2}\left(\Omega, \mathcal{F}_{T}, P\right)$.

We write $\Pi_{0}=\mathbb{R}, \Pi_{n}=\left\{\int_{\Delta_{n}(T)} f(r) d^{n} W(r) \mid f \in L^{2}\left(\Delta_{n}(T)\right)^{n d}\right\}$ for $n \in \mathbb{N}^{+}$(this space is called $n$-th Wiener chaos). The following theorem is well known (see, e.g., [4]).

Theorem 2.3 Take $\left(\mathcal{F}_{t}\right)_{t}$ as the natural completed Brownian filtration. Then $L^{2}\left(\Omega, \mathcal{F}_{T}, P\right)$ has the following orthogonal decomposition (called Wiener chaos decomposition): $L^{2}\left(\Omega, \mathcal{F}_{T}, P\right)=\oplus_{n=0}^{\infty} \Pi_{n}$.

From now on, $\left(\mathcal{F}_{t}\right)_{t}$ will be the natural completed Brownian filtration.

The main idea is the following. The stochastic (standard) integral acts like a shift for the Wiener chaos, i.e. formula (4). Then, if $u$ is a solution of (1), $Q_{n} u$ solves an equation which is (2) but for the stochastic part, which is driven by $Q_{n-1} u$ and thus can be regarded as a random external force, fixed a priori by inductive hypothesis. So the equation for $Q_{n} u$ is morally the Kolmogorov equation for (2).

Lemma 2.4 Let $X$ be an $\left(\mathcal{F}_{t}\right)_{t}$-progressively measurable process, with values in $\mathbb{R}^{d}$, such that $E\left[\int_{0}^{T}|X(t)|^{2} d t\right]<$ $+\infty$. Let $Q_{n}$ be the projector on the $n$-th Wiener chaos. Then 


$$
Q_{n+1} \int_{0}^{T} X(t) d W(t)=\int_{0}^{T} Q_{n} X(t) d W(t)
$$

Proof: Straightforward.

Hypotheses 2.5 b in $L_{l o c}^{2}\left(\mathbb{R}^{d}\right)^{d}$ and $\operatorname{div} b$ is in $L_{l o c}^{2}\left(\mathbb{R}^{d}\right)$.

We now state the main result.

Theorem 2.6 Suppose $\left(\mathcal{F}_{t}\right)_{t}$ is the Brownian filtration. Suppose $u_{0}$ in $L^{2}$ (resp. $\left.L^{\infty}\right)$, suppose hypotheses (2.5) and suppose uniqueness in the class of weak $L^{2}$ (resp. $L^{\infty}$ ) solutions for Kolmogorov equation

$$
\frac{\partial v_{t}}{\partial t}+B v_{t}=\frac{1}{2} K v_{t}
$$

Then there is uniqueness for (1) in the class of weak $L^{2}\left(\right.$ resp. $\left.L^{\infty}\right)$ solutions adapted to $\left(\mathcal{F}_{t}\right)_{t}$.

Proof: Let $u$ be a solution of (1) with $u_{0}=0$. By Wiener chaos decomposition, it is enough to show $Q_{n} u \equiv 0$ for every $n \in \mathbb{N}$. We will prove it inductively.

Projecting equation (2) on the $n$-Wiener chaos, for lemma (2.4) we obtain for every $\phi \in C_{c}^{\infty}$

$$
\left\langle Q_{n} u_{t}, \phi\right\rangle=\int_{0}^{t}\left\langle Q_{n} u_{r},\left(\frac{1}{2} K^{*}-B^{*}\right) \phi\right\rangle d r-\sum_{k} \int_{0}^{t}\left\langle Q_{n-1} u_{r}, D_{k}^{*} \phi\right\rangle d W_{r}^{k},
$$

where we have posed $Q_{-1} \equiv 0$. By inductive hypothesis $Q_{n-1} u \equiv 0$, this equation becomes equation (5), which has by hypothesis uniqueness property among weak solutions. The proof in the $L^{2}$ case is complete.

If $u$ is an $L^{\infty}$ solution, we obtain, reasoning as above, that $Q_{n} u$ satisfies (5), but is not necessarily in $L^{\infty}$. However, $Q_{n} u_{t}$ is the iterated stochastic integral of a deterministic function $f_{t}$, so $\int_{\Delta_{n}(t)} f_{t}(s) g(s) d^{n} s$ is a weak $L^{\infty}$ solution of (5) for every $g \in L^{\infty}\left(\Delta_{n}(T)\right)$; thus $f \equiv 0$. We are done in the $L^{\infty}$ case.

Remark 1 In the $L^{\infty}$ case, the result is valid also for $b \in L_{\text {loc }}^{1}\left(\mathbb{R}^{d}\right)$ with $\operatorname{div} b \in L_{\text {loc }}^{1}\left(\mathbb{R}^{d}\right)$, since one can show that the integrals in the proof make sense under these hypotheses.

\section{The uniqueness result}

In order to exploit theorem (2.6), we want to find sufficient condition for uniqueness for equation (5). Hypotheses 3.1 b is in $L^{p}\left(\mathbb{R}^{d}\right)^{d} \cap L_{l o c}^{2}\left(\mathbb{R}^{d}\right)^{d}$, divb is in $L^{q}\left(\mathbb{R}^{d}\right) \cap L_{\text {loc }}^{2}\left(\mathbb{R}^{d}\right)$ for $\left.\left.\left.\left.p \in\right] d,+\infty\right], q \in\right] d / 2,+\infty\right]$. Lemma 3.2 Under hypotheses (3.1), equation (5) has uniqueness property in the class of weak $L^{\infty}$ solutions and in the class of weak $L^{2}$ solutions.

Proof: Extending formula (2) for kernels $\phi^{s, x}(r, y)=(2 \pi(s-r))^{-d / 2} \exp \left(-\frac{|x-y|^{2}}{2(s-r)}\right)$, we get, if $t<s$,

$$
\left\langle u_{t}, \phi_{t}\right\rangle=\left\langle u_{0}, \phi_{0}\right\rangle+\int_{0}^{t}\left\langle u_{r},(\operatorname{div} b) \phi_{r}\right\rangle d r+\int_{0}^{t}\left\langle u_{r}, b \cdot \nabla \phi_{r}\right\rangle d r .
$$

We fix $u_{0} \equiv 0$. In the $L^{\infty}$ case (the $L^{2}$ case being similar), the RHS in (7) is bounded by

$$
C\left(\|\operatorname{div} b\|_{L^{q}\left(\mathbb{R}^{d}\right)}+\|b\|_{L^{p}\left(\mathbb{R}^{d}\right)}\right) \int_{0}^{t}\left\|u_{r}\right\|_{L^{\infty}\left(\mathbb{R}^{d}\right)}(s-r)^{-\alpha} d r,
$$

with $\alpha=\max \{d /(2 q),(1+d / p) / 2\} \in] 0,1[$ (since $p>d$ and $q>d / 2)$. Now we let $s \rightarrow t$, so that $\left\|u_{t}\right\|_{L^{\infty}}$ is bounded by (8) (with $s$ replaced by $t$ ). We can conclude using classical Gronwall arguments. 
Corollary 3.3 Under hypotheses (3.1), the STLE (1) has uniqueness property in the class of weak $L^{2}$ solutions adapted to $\left(\mathcal{F}_{t}\right)_{t}$ and in the class of weak $L^{\infty}$ solutions adapted to $\left(\mathcal{F}_{t}\right)_{t}$.

Remark 2 Existence for such solutions is proved at least in the class of weak $L^{\infty}$ solutions, under more general hypotheses, namely $b \in L_{\text {loc }}^{1}\left([0, T] \times \mathbb{R}^{d} ; \mathbb{R}^{d}\right)$, div $b \in L_{\text {loc }}^{1}\left([0, T] \times \mathbb{R}^{d}\right)([q])$.

Remark 3 In the $L^{\infty}$ case, corollary (3.3) works also for $b \in L^{\infty}\left([0, T] ; L^{p}\left(\mathbb{R}^{d}\right)^{d}\right)$, $\operatorname{div} b \in L^{\infty}\left([0, T] ; L^{q}\left(\mathbb{R}^{d}\right)\right)$, for some $p>d, q>d / 2$, with a similar proof and using remark (1). The corresponding deterministic result does not hold: a counterexample is due to Depauw ([5], even if uniqueness holds in a smaller class, see [2]). For this and the following example, also previous results on regularization by noise do not apply.

Remark 4 Another counterexample can be adapted from the example in [6], at pages 541-543. More precisely, the drift $b$ is generated on a bounded ball by the following Hamiltonian: $H\left(x_{1}, x_{2}\right)=-x_{1} /\left|x_{2}\right|^{1 / 2}$ if $\left|x_{1}\right| \leq\left|x_{2}\right|^{1 / 2}, H\left(x_{1}, x_{2}\right)=-x_{1}+\operatorname{sgn}\left(x_{1}\right)\left[\left|x_{2}\right|^{1 / 2}-1\right]$ if $\left|x_{1}\right|>\left|x_{2}\right|^{1 / 2}$. It can be shown, as in [6], that $b \in L_{l o c}^{1}$ with $\operatorname{div} b=0$ and that the corresponding equation has more than one solution. In the stochastic case uniqueness is restored, even if $b$ does not satisfy hypotheses (3.1): one can prove an estimate like (8), using the scaling property $|b(\beta x)|=|\beta|^{-1 / 2}|b(x)|$ if $\left|x_{1}\right| \leq\left|x_{2}\right|^{1 / 2}$.

Remark 5 Existence and pathwise uniqueness have been proved for the $S D E d X_{t}=b\left(X_{t}\right)+d W_{t}$, when $b$ is only in $L^{p}\left(\mathbb{R}^{d}\right)$ for some $p>d([8])$. However, we do not know a way to exploit this result to obtain uniqueness for weak solutions of STLE. Indeed, the relation $u_{t}\left(X_{t}\right)=u_{0}$ holds with some regularity hypotheses on u (remark (4) gives a deterministic counterexample). Furthermore, the hypothesis $\operatorname{div} b \in$ $L_{l o c}^{1}$ is needed to give sense to definition (2.1) and some integrability assumptions on divb are required in many articles about the topic ([1], [6], [3], [7]). Nevertheless, some nontrivial links between SDE and STLE could be possible; we plan to analyze it in a forthcoming paper.

\section{Acknowledgements}

I am indebted to Prof. F. Flandoli for the idea of proof of lemma (3.2); he also gave me suggestions about this article and in general about STLE, Le Jan - Raimond article and their links. I also thank Prof. L. Ambrosio for a discussion about the deterministic counterexamples.

\section{References}

[1] L. Ambrosio, Transport equation and Cauchy problem for $B V$ vector fields, Invent. Math. 158 (2004), 227-260.

[2] L. Ambrosio, G. Crippa, A. Figalli, L.V. Spinolo, Some new well-posedness results for continuity and transport equation, and applications to the chromatography system, SIAM J. Math. Anal. 41 (2009), 1890-1920.

[3] S. Attanasio, F. Flandoli, Renormalized solutions for stochastic transport equations and the regularization by bilinear multiplicative noise, arXiv:1007.4102.

[4] N. Bouleau, F. Hirsch, Dirichlet forms and analysis on Wiener space, de Gruyter, Berlin, 1991.

[5] N. Depauw, Non unicité des solutions bornées pour un champ de vecteurs $B V$ en dehors d'un hyperplan, C. R. Acad. Sci. Paris, Ser. I 337 (2003), 249-252.

[6] R.J. DiPerna, P.-L. Lions, Ordinary differential equations, transport theory and Sobolev spaces, Invent. Math. 98 (1989), 511-547.

[7] F. Flandoli, M. Gubinelli, E. Priola, Well-posedness of the transport equation by stochastic perturbation, Invent. Math. 180 (2010), 1-53.

[8] N.V. Krylov, M. Röckner, Strong solutions of stochastic equations with singular time dependent drift, Probab. Theory Relat. Fields 131 (2005), 154-196.

[9] Y. Le Jan, O. Raimond, Integration of Brownian vector fields, Ann. Probab. 30 (2002), 826-873. 